sichtsbahn. Störungen in der Nähe des Anflugspunktes wurden regelmäßig damit beantwortet, daß das Hummelmännchen „Gold“ den visuell ver änderten Anflugspunkt einfach überging und sogleich den nächsten Punkt anflog.

Dennoch handelt es sich bei den Flugbahnen der Hummelmännchen vorwiegend um Geruchsbahnen. Das geht auch daraus hervor, daß Hummelmännchen „Gold“ fast täglich seine Bahn in den Morgenstunden bei den ersten Flügen neu mit Duftstoffen versah. Es ist hier wohl eine kombinierte Tätigkeit der beiden Hauptsinne, des Geruches und des Gesichtes, anzunehmen. Einige Beobachtungen deuten auch darauf hin, daß den Männchen bei ihrem Bahnflug ein ausgebildetes Registriervermögen für bestimmte Strecken, Flugwinkel und bestimmte Zeiten zukommt, worüber in einer späteren Arbeit berichtet werden soll.

\title{
Der Ölgehalt verschiedenchromosomiger Rassen vom Kalmus (Acorus Calamus L.) .
}

\author{
Von Heinz Diedrich WulfF \\ Aus dem Botanischen Institut der Universität Kiel \\ (Z. Naturforschg. 1, 600-603 [1946]; eingegangen am 8. April 1946)
}

\begin{abstract}
Der Gehalt an ätherischem öl wurde bei drei verschiedenen Chromosomenrassen von Acorus Calamus $L$, bestimmt und die folgenden Werte gefunden: Diploide Rasse $(2 n=24) 2,17 \%$, triploide Rasse $(2 n=36) 3,12 \%$, tetraploide Rasse $(2 n=48) 6,82 \%$. Somit nimmt der Gehalt an ätherischem Öl mit steigender Chromosomenzahl zu. Hinsichtlich des Wassergehaltes ergeben diese drei Rassen keine statistisch gesicherte Differenz. Vergleichsweise wurden die Untersuchungen auch auf Acorus gramineus Sol. ausgedehnt. Der Ölgehalt dieser diploiden Art liegt bei $0,7 \%$, ohne daß eine grünblättrige und eine variegate Form Unterschiede zeigten.
\end{abstract}

$I^{n}$ n einer früheren Veröffentlichung ${ }^{1}$ habe ich nachgewiesen, daß beim Kalmus drei Chromosomenrassen vorkommen, nämlich eine diploide mit $2 n=24$, eine tetraploide mit $2 n=48$ und eine triploide mit $2 n=36$ Chromosomen. Diese letztere ist die vor allem in der nördlichen Hemisphäre weitverbreitete sterile Rasse, die nur zu vegetativer Vermehrung befähigt ist, da es infolge der Triploidie nicht zur Bildung funktionsfähiger Samenanlagen und Pollenkörner kommen kann. Ihre Chromosomenzahl $2 n=36$ wurde von $\mathrm{O}$. $\mathrm{Palmg} \mathrm{ren}{ }^{2}$ auch für wildwachsende Kalmuspflanzen Schwedens ermittelt. Die untersuchten diploiden und tetraploiden Pflanzen habe ich dagegen aus Samen herangezogen, die aus den Botanischen Gärten in Kopenhagen bzw. Leningrad stammten. Uber das Wildvorkommen dieser beiden Rassen können zur Zeit keine über bloße Vermutungen hinausgehenden Ansichten geäußert werden. Außer den drei Rassen von Acorus Cala-

1 Planta 31, 478 [1941]

= Bot. Not. 1943. 348 . mus $L$. ist weiterhin der habituell stark abweichende Acorus gramineus Sol. durch die Zählung G. Nakajima s${ }^{3}$, die ich bestätigen konnte, chromosomal als diploid bekannt gemacht worden $(2 n=24)$. Aus der aufgeführten orthoploiden Reihe der Chromosomenzahlen fällt allerdings der in Nordamerika einheimische fertile Kalmus heraus. Nach Untersuchungen von $\mathrm{D}$ a h $\mathrm{l}$, die von M. G. D u d l e $y^{4}$ und M. F. B u e $11^{5}$ zitiert werden, besitzt diese interessante Kalmusrasse nur $2 n=18$ Chromosomen. Es wird notwendig sein, sie späterhin vergleichsweise noch eingehender zu studieren.

Ohne damit eine prinzipielle Stellungnahme ausdrücken zu wollen, hatte ich mich in meiner früheren Arbeit der landläufigen Ansicht angeschlossen, daß die Ahnen der heute in Westeuropa heimisch gewordenen Kalmuspflanzen aus kleinasiatischen Standorten über Konstantinopel-Wien eingeführt worden seien. Für Osteuropa wurde daneben bislang noch

3 Jap. J. Gen. 9, 1 [1933].

4 Bot. Gaz. 98, 556 [1937].

s Bot. Gaz. 99, 556 [1938]. 
eine Einführung oder Einschleppung durch die Tataren für möglich gehalten. Dieser Ansicht widerspricht $\mathrm{K}$. We i ${ }^{6}$ in einer Arbeit, von der ich erst nach Drucklegung meiner früheren Veröffentlichung Kenntnis erhielt, sehr lebhaft. Er vertritt den Standpunkt, daß eine zweimalige Einführung nicht anzunehmen sei, sondern daß die Pflanze sowohl nach West- als auch nach Osteuropa (Polen) ihren Weg über Konstantinopel-Wien genommen habe. Außerdem soll der ursprüngliche Herkunftsort der ersten eingeführten Pflanzen nicht in Kleinasien zu suchen sein, wie man bisher allgemein annahm, sondern in Indien. Diese von $\mathrm{W}$ e in vertretenen Korrekturen an unserer bisherigen Auffassung über die Einführungsgeschichte des Kalmus dürften wohl allgemeinere Beachtung verdienen. Daß auch der Kalmus Indiens, wie ich bereits an Pflanzen aus Annamalainagar nachwies, triploid ist, sei in diesem Zusammenhang nochmals betont.

Nachdem zunächst die aus allgemeinbiologischem Interesse bemerkenswerte Sterilität unseres Kalmus ihre Aufklärung gefunden hatte; mußte natürlich das Vorhandensein der verschiedenen Chromosomenrassen im Hinblick auf die sehr umfangreich gewordene Polyploidieforschung einen starken Anreiz zu vergleichenden Untersuchungen über ihre physiologischen Leistungen und ihre morphologische Ausgestaltung geben. Von den in diesem Zusammenhang denkbaren Problemen seien an dieser Stelle einige Beobachtungen über den Gehalt an ätherischem öl mitgeteilt. Sämtliche Ölbestimmungen wurden mit der von O. M o r it z beschriebenen Apparatur ${ }^{7}$ durchgeführt, für deren leihweise Überlassung ich Hrn. Prof. Moritz auch an dieser Stelle bestens. danke.

Nach einigen ermutigenden Stichproben im Herbst 1941 wurden im Oktober-November 1942 weitere Rhizomstücke gesammelt und getrocknet, und zwar von den in Tab. 1 genannten, im Botanischen Garten Kiel in Kultur befindlichen Pflanzen, die, alle in kleine Kübel gepflanzt, in Beete eingebettet und gut feucht gehalten, unter möglichst gleichartigen Kulturbedingungen standen.

Bei Durchführung der insgesamt 43 Analysen zeigte sich als erstes, statistisch erhärtetes Ergebnis (stets $P<0,0027)^{8}$, daß der Ölgehalt bei dem Material gleicher Polyploidiestufen von Acorus Calamus sich innerhalb der gleichen Größen bewegte, so daß für eine weitere rechnerische Auswertung'nur die drei verschiedenen Polyploidie-

${ }^{6}$ Hercynia 1, 367 [1939].

7 Arch. Pharmaz. Ber. dtsch. pharmaz. Ges. 1938, $368 \mathrm{ff}$.

\begin{tabular}{|c|c|c|c|}
\hline Untersuchte Pflanzen & Herkunft & 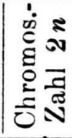 & $\begin{array}{c}\text { Anzahl } \\
\text { der } \\
\text { Ana- } \\
\text { lysen }\end{array}$ \\
\hline 1. Acorus gramineus Sol. & $\begin{array}{l}\text { Straßburg } \\
\text { (grünblättrig) }\end{array}$ & 24 & 2 \\
\hline 2. Acorus gramineus Sol. & $\begin{array}{l}\text { Kiel (variegate } \\
\text { Form) }\end{array}$ & 24 & 2 \\
\hline 3. Acorus Calamus L. & $\begin{array}{l}\text { Kopenhagen } \\
\text { (alte Pflanze) }\end{array}$ & 24 & $\tilde{\mathbf{5}}$ \\
\hline 4. Acorus Calamus L. & $\begin{array}{l}\text { Kopenhagen } \\
\left(\text { Sämling } \mathrm{K}_{4}\right)\end{array}$ & 24 & 5 \\
\hline 5. Acorus Calamus $L$. & Schwabstedt & 36 & 10 \\
\hline 6. Acorus Calamus $L$. & Kiel & 36 & 5 \\
\hline 7. Acorus Calamus L. & $\begin{array}{l}\text { Leningrad } \\
\left(\text { Sämling } L_{1}\right)\end{array}$ & 48 & 9 \\
\hline 8. Acorus Calamus $L$. & $\begin{array}{l}\text { Leningrad } \\
\quad\left(\text { Sämling } \mathrm{L}_{2}\right)\end{array}$ & 48 & 5 \\
\hline
\end{tabular}

Tab. 1. Übersicht über das Untersuchungsmaterial.

grade berücksichtigt zu werden brauchten. Ebenso ergab sich kein Unterschied im ölgehalt des getrockneten Rhizoms für die grünblättrige und die variegate Form von Acorus gramineus. Weiterhin wurden die in Tab. 2 zusammengestellten Werte errechnet.

\begin{tabular}{|c|c|c|}
\hline Untersuchte Pflanzen & $\begin{array}{c}\text { Mittelwert }{ }^{9} \text { des } \\
\text { Ölgehalts von } 3 \mathrm{~g} \\
\text { getrocknetem } \\
\text { Rhizom }\end{array}$ & $\begin{array}{l}\text { Durch- } \\
\text { schnittl.öl- } \\
\text { gehalt des } \\
\text { getrock- } \\
\text { neten Rhi- } \\
\text { zoms in \% }\end{array}$ \\
\hline 1. Acorus gramineus Sol. & $0,0209 \pm 0,0005$ & 0,7 \\
\hline $\begin{array}{l}\text { 2. Acorus Calamus } L \text {. } \\
\text { (diploid) }\end{array}$ & $0,0652 \pm 0,0047$ & 2,17 \\
\hline $\begin{array}{l}\text { 3. Acorus Crilamus } L \text {. } \\
\text { (triploid) }\end{array}$ & $0,0936 \pm 0,0015$ & 3,12 \\
\hline $\begin{array}{l}\text { 4. Acorus Calamus } L \text {. } \\
\text { (tetraploid) }\end{array}$ & $0,2047 \pm 0,0110$ & 6,82 \\
\hline
\end{tabular}

Tab. 2. Ölgehalt der verschiedenen Arten bzw. Rassen.

Eine Berechnung der Differenzen der Mittelwerte führte sodann $\mathrm{zu}$ den in Tab. 3 angegebenen Werten.

Aus einer Beurteilung der Mittelwerte und ihrer mittleren Fehler ergibt sich, daß in allen Fällen in

8 K. Pät a u, Zur statistischen Beurteilung von Messungsreihen. (Eine neue $t$-Tafel.) Biol. Zbl. 63, 152 [1943].

9 Es ist in den Tabellen stets der einfache mittlere Fehler angegeben. 


\begin{tabular}{|c|c|}
\hline Gebildete Differenzen & $\begin{array}{c}\text { Differenz } \\
\text { der Mittelwerte }\end{array}$ \\
\hline $\begin{array}{c}\text { 1. Acorus Calamus triploid / Acorus } \\
\text { Culamus diploid }\end{array}$ & $0,0284 \pm 0,0035$ \\
$\begin{array}{c}\text { 2. Acorus Calamus tetraploid / Aco- } \\
\text { rus Calamus triploid }\end{array}$ & $0,1111 \pm 0,0107$ \\
$\begin{array}{c}\text { 3. Acorus Calamus diploid / Acorus } \\
\text { gramineus }\end{array}$ & $0,0443 \pm 0,0076$ \\
\hline
\end{tabular}

Tab. 3. Differenzen der Mittelwerte für den ölgehalt.

bezug auf den ölgehalt trotz der verhältnismäßig geringen Analysenzahl bei den untersuchten Rassen von Acorus Calamus bzw. zwischen dem diploiden Acorus Calamus und Acorus gramineus ein statistisch gesicherter Unterschied besteht $(P$ stets wesentlich kleiner als der „kritische“ Wert 0,0027). Innerhalb der zur Beobachtung stehenden Grenzen ist somit bei Acorus Calamus mit dem Ansteigen der Chromosomenzahl eine Steigerung des Gehaltes an ätherischem Öl verbunden.

Daß die von mir beobachteten Werte des Ölgehaltes verhältnismäßig gut mit den Angaben in der Literatur übereinstimmen, erweist die in der Tab. 4 gebrachte Zusammenstellung.

\begin{tabular}{|c|c|c|}
\hline \multirow{2}{*}{ Untersuchte Pflanzen } & \multicolumn{2}{|c|}{$\begin{array}{c}\text { Ölgehalt des getrockneten } \\
\text { Rhizoms in } \%\end{array}$} \\
\hline & $\begin{array}{c}\text { nach } \\
\text { W e h m e r } \\
(19.9)\end{array}$ & $\begin{array}{l}\text { nach meinen } \\
\text { Analysen }\end{array}$ \\
\hline 1. Acorus gramineus Sol. & $0,5-0,9$ & 0,7 \\
\hline & & $\begin{array}{c}2,17 \\
\text { (diploid) }\end{array}$ \\
\hline 2. Acorus Calamus L. & $1,5-3,5$ & $\begin{array}{c}3,12 \\
\text { (triploid) }\end{array}$ \\
\hline 3. Acorus Calamus L. & - & $\begin{array}{c}6,82 \\
\text { (tetraploid) }\end{array}$ \\
\hline 4. Acorus spurius Schntt & his 5 & - \\
\hline
\end{tabular}

Tab. 4. Vergleich der Angaben über den Ölgehalt der Gattung Calamus.

Besonders schön ist die Ubereinstimmung der Werte bei Acorus gramineus und dem triploiden Acorus Calamus. Außerdem dürfen wir vielleicht vermuten, daß in Unkenntnis der chromosomalen Gegebenheiten in dem von $\mathrm{C}$. W e h m e r ${ }^{10}$ zitierten Wert von 1,5 bis $3,5 \%$ auch Untersuchungen über die diploide Rasse mitenthalten sind. Un-

10 Die Pflanzenstoffe, 2. Aufl., Bd. 1, Jena 1929. klarer liegen die Verhältnisse hinsichtlich des Acorus spurius Schott. Im allgemeinen wird die Gattung Acorus, so u.a. auch von Engle r ${ }^{11}$, nur in die Arten A.gramineus und A.Calamus unterteilt, von der die letztere vier Varietäten, darunter die Varietät spurius Schott, enthält. Diese, der ,,japanische Kalmus“, wird jedoch gelegentlich auch als besondere Art geführt. Immerhin erwähnt ebenfalls Wehmer noch anmerkungsweise, daß es nicht sicher sei, ob es sich hier um eine besondere Art handle, und daß das Öl von dem des gewöhnlichen Kalmus verschieden sei, da Asahina (1906) fand, daß es linksdrehend, das des gewöhnlichen Kalmus aber rechtsdrehend ist. Dazu sei zunächst nur bemerkt, daß nach meiner Beobachtung alle vier Rhizomund Ölproben Differenzen im Geruch zeigten. Es ist also möglich, daß mit der Polyploidisierung nicht nur quantitative, sondern auch qualitative Unterschiede aufgetreten sind.

Auch W e i n führt den ,japanischen Kalmus“, A. spurius Schott, nur als Varietät von A.Calamus an. Er erwähnt ausdrücklich, was in diesem Zusammenhang besonders interessiert, daß Fruchtbildung beobachtet sei. Acorus spurius kommt von Japan über Sibirien bis nahe an das europäische Rußland vor, und da ich die Samen der zu. meinen Untersuchungen herangezogenen Pflanzen gerade aus dem Botanischen Garten in Leningrad erhielt, ist die Vermutung naheliegend, daß die Varietät spurius mit meiner tetraploiden Rasse identisch sein könnte. Ob diese Vermutung, für die neben dem hohen Ölgehalt bisher vor allem die. Fertilität und die Blattbreite sprechen, zu Recht besteht, bedarf natürlich noch eingehenderer Feststellungen, die aber unter den augenblicklichen Verhältnissen nicht durchführbar sind. Leider ließ sich auch noch nicht ermitteln, ob das öl der tetraploiden Rasse etwa wie das des A.spurius links dreht, da die dafür bereitgestellten Ölproben durch Fliegerschaden verloren gingen.

Mit dem Ölgehalt kam zwangsläufig auch derWassergehalt der Rhizome zur Beobachtung, weil diese jeweils bis zur Gewichtskonstanz getrocknet wurden. Die im Herbst der Jahre 1941 und 1942 erhaltenen Werte zeigt Tab. 5.

Wie diese Tabelle zeigt, sind gesicherte Unterschiede im Wassergehalt der beiden Arten, bzw.

11 A. Engler, Araceae. In A. Engler u. K. Prantl, Die natürlichen Pflanzenfamilien, Tl. 2, Leipzig 1889. 


\begin{tabular}{|c|c|c|c|}
\hline Untersuchte Pflanzen & $\begin{array}{c}\text { Mittlerer } \\
\text { Wassergehalt } \\
\text { des Rhizoms } \\
\text { in } \%\end{array}$ & 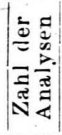 & $\begin{array}{c}\text { Differenz } \\
\text { der } \\
\text { Mittelwerte }\end{array}$ \\
\hline $\begin{array}{l}\text { 1. Acorus gramineus } \\
\text { 2. Acorus Calamus } \\
\text { (diploid) } \\
\text { 3. Acorus Calamus } \\
\text { (triploid) } \\
\text { 4. Acorus Culamus } \\
\text { (tetraploid) }\end{array}$ & $\begin{array}{l}59,30 \pm 3,04 \\
62,53 \pm 0,58 \\
62,15 \pm 1,38 \\
57,93 \pm 2,88\end{array}$ & $\begin{array}{l}2 \\
4 \\
4 \\
4\end{array}$ & $\begin{array}{l}3,23 \pm 3,09 \\
0,38 \pm 1,49 \\
4,22 \pm 3,19\end{array}$ \\
\hline
\end{tabular}

Tab.5. Der Wassergehalt der untersuchten Kalmusarten bzw. -rassen

der drei Rassen, nicht vorhanden. Allerdings ist die Zahl der Analysen nur gering gewesen. Doch auch im Frühjahr 1944 getrocknetes Material der diploiden und der tetraploiden Rasse von Acorus Calamus brachte mit $62,26 \%$ für die erstere und
$62,75 \%$ Wassergehalt für die letztere Rasse keine ausgeprägten Differenzen.

Im Vergleich namentlich mit künstlich hergestellten Autopolyploiden ist das Konstantbleiben des Wassergehaltes besonders bemerkenswert, werden diese doch häufig nur als ,,aufgeschwemmte" Diploide betrachtet. Ihnen gegenüber dürfen wir in den verschiedenen Rassen von Acorus Calamus wohl mit Recht natürliche Polyploide, und zwar Autopolyploide, sehen, denn bei der geringen Artenzahl innerhalb der Gattung läßt sich die Annahme einer Allopolyploidie kaum vertreten. Im ganzen gesehen, ergibt sich nach dem Vorstehenden eine deutliche Überlegenheit der polyploiden Formen, welche die allgemeine Bedeutung und die Notwendigkeit der Untersuchung wild vorkommender, von der Natur bereits ausgelesener Polyploider für eine eventuelle züchterische Weiterbearbeitung zu unterstreichen geeignet ist.

\section{MITTEILUNGEN}

\section{Das Astronomentreffen in Hamburg-Bergedorf am 26. - 28. September 1946}

$\mathrm{S}$ ieben Jahre sind seit der letzten Tagung der Astronomischen Gesellschaft (A.G.) in Danzig verflossen, und fünf Jahre seit dem letzten Kolloquium der deutschen Astronomen in Göttingen. Die A. G. hatte internationalen Charakter und mußte ihre Tätigkeit während des Krieges einstellen. Heute steht die deutsche Astronomie vor der Notwendigkeit, die $\mathrm{Zu}$ sammenarbeit der deutschen Sternwarten in einem bescheideneren Rahmen zu organisieren. Dieses war die wichtigste Aufgabe des Hamburger Treffens der Direktoren der deutschen Sternwarten, an dem als Gäste die Engländer Sir S p en c e r - J o n e s (Astronomer Royal) und Colonel S m ith, beide aus Preenwich, teilnahmen. Leider waren die Vertreter aus der russischen Besatzungszone, die alle zugesagt hatten, aus unbekannten Gründen nicht erschienen. Die Vertreter der Institute in Berlin-Neubabelsberg, Potsdam, Jena und Greifswald fehlten, die Sternwarte Leipzig ist vollkommen zerstört, Königsberg und Breslau liegen jetzt außerhalb des deutschen Kulturgebietes. Man kann trotzdem hoffen, daß die Beschlüsse der Institute der westlichen Zone (Bonn, Göttingen, Hamburg, Heidelberg Recheninstitut, Heidelberg Königstuhl, Freiburg, Kiel und München) für die gesamte deutsche Astronomie bindend bleiben werden.

Nachdem die auswärtigen Vorstandsmitglieder der A. G. ihren Rücktritt mitgeteilt hatten, wobei sie aus- drücklich ihrem Wunsche Ausdruck gaben, daß die Gesellschaft als deutsche Gesellschaft fortbestehen und ihre großen Aufgaben fortsetzen möge, wurde beschlossen, diesem Wunsche stattzugeben und ohne wesentliche Änderungen der Statuten, nur mit verkleinertem Vorstande, die Gesellschaft bestehen zu lassen und alle ihre großen Gemeinschaftsarbeiten fortzusetzen. Die Vertreter der Sternwarten berichteten über den Zustand ihrer Institute und die Möglichkeit, die begonnenen Gemeinschaftsarbeiten fortzusetzen und neue zu unternehmen. Erfreulicherweise zeigte es sich, daß die durch den Krieg erlittenen Schäden im allgemeinen nicht bedeutend waren, daß mehrere Institute sich im Kriege sogar noch erweitern konnten und daß sogar die Sternwarte München, trotz der großen Schäden an Wohngebäuden, dank der energischen Aufbauarbeit in Kürze voll einsatzfähig sein wird. Als Neugründungen, die während des Krieges entstanden, sind vor allem das Fra un of e r Institut in Freiburg mit den Sonnenwarten auf dem Wendelstein und auf dem Schauinsland $\mathrm{zu}$ nennen, deren Aufgabe es ist, die Sonne mit Hilfe von Spektroheliographen und Koronographen auf Flecken, Fackeln, Protuberanzen und Korona zu überwachen und den Zusammenhang dieser Erscheinungen mit Störungen in der Ionosphäre der Erde sowie Polarlichtern und magnetischen Stürmen zu studieren. Auch die Sternwarten Göttingen und Hamburg haben 